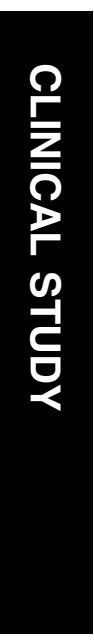

\title{
Laser-induced chorioretinal venous anastomosis using combined lasers with different wavelengths
}

Ophthalmology Center of Beijing Tong-Ren Hospital, Capital Medical University, Beijing, China

Correspondence: NL Wang, Ophthalmology Center of Beijing Tong-Ren Hospital, Capital Medical University, Dong Jiao Min Xiang No.1, Beijing 100730,

China

Tel: + 861058268251

Fax: + 86065131244 .

E-mails: plf@public3.bta. net.cn and luningnl@yahoo. com.cn

Received: 20 September 2005

Accepted in revised form: 21 March 2006

Published online: 19 May 2006

Ethic approval: Beijing Committee Review Board approval was obtained at the beginning of the study. The location of the Committee Review Board is Beijing Health Bureau

Competing interest statement: There are no proprietary or commercial interests

Previous presentation: This study was partly presented in the First Seri-ARVO meeting on research in vision and ophthalmology in Singapore in 2003, hosted by Singapore Eye Research Institute and ARVO

\begin{abstract}
Aim To evaluate the effectiveness of combined lasers with different wavelengths in creating chorioretinal venous anastomosis (CRVA) in nonischaemic central retinal vein occlusion (CRVO).

Methods In this retrospective, noncomparative, consecutive case series, records of all patients with a diagnosis of nonischaemic CRVO who underwent combined laser treatment to induce CRVA and who were followed-up for 6 months or longer were reviewed.

Results Records of 85 patients ( 85 eyes) were analysed. These patients were divided into two groups. In group 1 (24 cases, 24 eyes), combined lasers with two different wavelengths (argon blue-green laser combined with Nd-YAG laser were used. In group 2 (61 cases, 61 eyes), combined lasers with three different wavelengths (argon bluegreen laser combined with krypton red laser and Nd-YAG laser) were used. Functional anastomosis formed in 13 out of 24 eyes (54\%) in group 1 and 47 out of 61 eyes (77\%) in group 2. In patients with successful anastomosis, visual acuity improved for two or more lines in 49 out of 60 eyes $(82 \%)$, with the remaining eyes undergoing no change. There were no serious complications.

Conclusion The use of three laser wavelengths results in the creation of laser anastomosis and the success rate may be higher than when using two wavelengths alone.

Eye (2007) 21, 962-967; doi:10.1038/sj.eye.6702362; published online 19 May 2006
\end{abstract}

Keywords: chorioretinal venous anastomosis (CRVA); nonischaemic central retinal vein
N Lu, NL Wang, ZH Li, GL Wang, F Zhang

and $X Y$ Peng

occlusion (CRVO); argon laser; krypton red laser; Nd-YAG laser

Introduction

Laser-induced chorioretinal venous anastomosis (CRVA) was shown to be a new and effective treatment for retinal vein occlusion. ${ }^{1,2}$ A high-intensity argon laser was applied to a vein and underlying or adjacent Bruch's membrane, sometimes combined with an Nd:YAG laser, to induce the anastomosis formation. But there were two problems that limited its use in the clinic: (1) the success rate of anastomosis formation was relatively low (33-54\%) and (2) it was sometimes associated with serious complications. The most serious complications associated with the treatment were choroidovitreal neovascularization that may lead to vitreous haemorrhage and traction retinal detachment. ${ }^{1-6}$

We describe the treatment results using combined lasers with different wavelengths to induce CRVA in 85 eyes of 85 consecutive patients with nonischaemic central retinal vein occlusion (CRVO).

Patients and methods

Patients

Records of all patients with nonischaemic CRVO treated with laser-induced CRVA between 1999 March and 2004 October were reviewed retrospectively. Nonischaemic CRVO was determined to be less than 10-disc diameters of capillary nonperfusion seen on fluorescein angiography in the area corresponding to that used by the Central Vein 
Occlusion Study. ${ }^{7}$ As neovascular complications were associated with retinal ischaemia, ${ }^{1}$ we selected patients with capillary nonperfusion of less than 5-disc diameters, in order to decrease the occurrence of neovascular complications. All the patients had experienced a progressive deterioration of visual acuity for at least 1 month. Informed consent with full disclosure of the experimental nature of the treatment was obtained from all the patients. Committee Review Board approval for the treatment was obtained at the beginning of the study.

\section{Treatments}

With the use of topical anaesthesia and fundus contact lens, laser photocoagulation was applied to selected locations along a retinal vein, at least 3-disc diameters superior or nasal to the optic disc. Combined lasers with different wavelengths were used. Before 2000 February, combined lasers with two different wavelengths (argon blue-green laser combined with Nd-YAG laser) were used in all the cases. The argon blue-green laser (2-2.4 W, $50 \mu \mathrm{m}, 0.1 \mathrm{~s})$ was applied at the edge or on top of the vein, usually one, and occasionally two shots at each treatment site. Then the Nd-YAG laser (3-8 mj) was applied at the treatment site to break the retinal vein. From 2000 February, combined lasers with three different wavelengths (argon blue-green laser combined with krypton red laser and Nd-YAG laser) were used. At first, the argon blue-green laser (1.6-2 W, $50 \mu \mathrm{m}, 0.1 \mathrm{~s})$ was applied at the edge or on top of the vein, usually one, and occasionally two shots at each treatment site. Then, the krypton red laser $(1 \mathrm{~W}, 50 \mu \mathrm{m}, 0.1 \mathrm{~s})$ was applied at the same site, usually one, and occasionally two shots at each treatment site. At last, the $\mathrm{Nd}-\mathrm{YAG}$ laser (3-8 mj) was applied at the treatment site to break the retinal vein. Localized retinal haemorrhage at the treatment site could be controlled with pressure on the contact lens. All the treatments were completed by one doctor.

Scattered argon laser photocoagulation (argon green laser, $0.16-0.20 \mathrm{~W}, 500 \mu \mathrm{m}, 0.2 \mathrm{~s}$ ) was applied over the drainage area of the vein distal to the treatment sites for all the cases. Usually, 400-600 laser sports were applied.

The patients were examined 2 weeks after treatment, and then at monthly intervals until anastomosis formed. After that, the patients were followed at 3 months interval. The criterion to determine a successful anastomosis (Figures 1-3) was the ophthalmoscopic appearance of the central/peripheral segment of the treated vein dipping down into the choroid at the treatment site and preferential filling of the anastomotic vein at the early stage of fundus fluorescein angiography (FFA). Post-treatment fundus photographs and FFA were obtained. During follow-up, progression of nonischaemic CRVO to the ischaemic type was defined by the

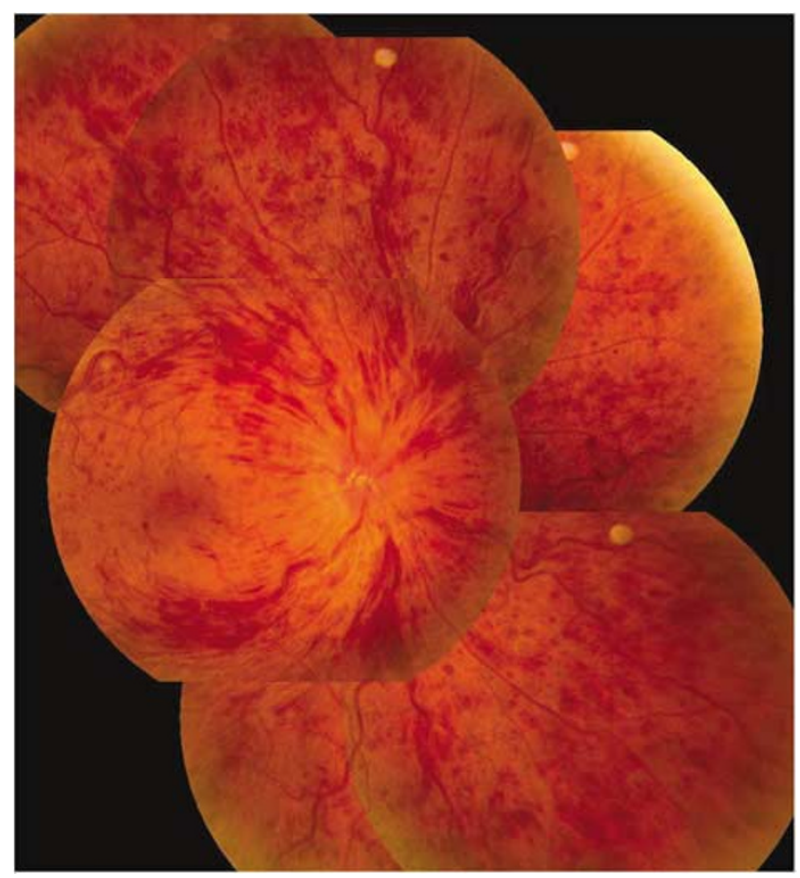

Figure 1 Coloured fundus photograph of right eye before treatment.

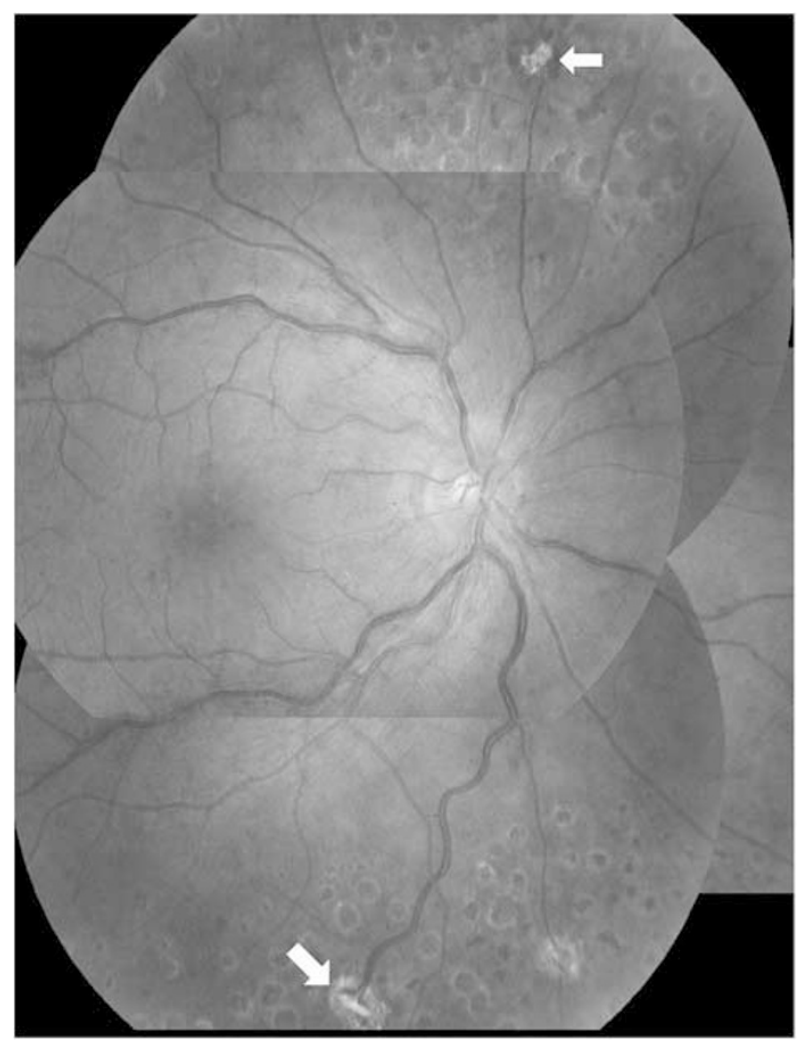

Figure 2 Coloured fundus photograph of the same eye 3 months after treatment. Successful anastomosis formed inferiorly, the retinal vein was broken and dipped down into the choroid (arrow). 


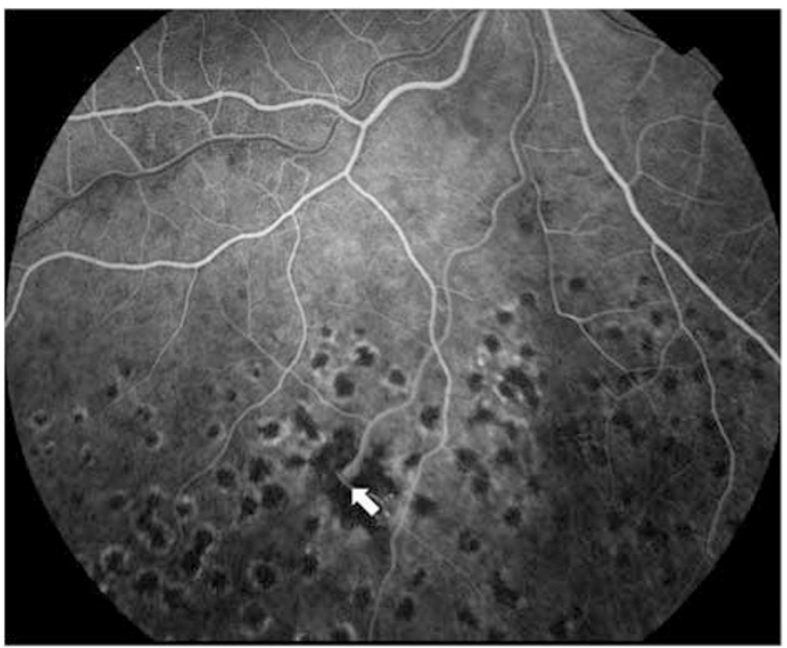

Figure 3 Early stage of fundus fluorescein angiography of the same eye 3 months after treatment, demonstrating preferential filling of the anastomotic vein at the treatment site (arrow).

development of more than 10-disc areas of capillary nonperfusion on FFA. Visual acuity was measured with best correction on a Snellen chart by trained staff masked to the treatment.

The $\chi^{2}$ test was used to determine whether the proportions of a single group divided into two categories were significantly different, with a significance acceptance level of $P<0.05$. All mean data are expressed as a mean $\pm \mathrm{SD}$.

\section{Results}

A total of 85 patients ( 85 eyes) with nonischaemic CRVO received the treatments (Table 1). Ages varied from 18 to 70 years, with an average \pm SD of $51.0 \pm 12$.2. Patients who received the argon blue-green laser combined with the Nd-YAG laser treatment was called group 1 (24 cases, 24 eyes); patients who received the argon bluegreen laser combined with the krypton red laser and the $\mathrm{Nd}-\mathrm{YAG}$ laser treatment was called group 2 (61 cases, 61 eyes). In comparison, the age, duration of CRVO before treatment, and visual acuity at the time of treatment was not significantly different between the two groups $(P>0.05)$. Follow-up time ranged from 6 to 36 months (average \pm SD of $12.2 \pm 9.9$ months). Successful anastomosis formed in 13 out of 24 eyes $(54 \%)$ in group 1 and 47 out of 61 eyes (77\%) in group 2. The number of eyes with successful anastomosis was significantly different between the two groups $(P<0.05)$. Two to four treatment sites were selected for each eye, with a mean of $2.5 \pm 0.7$ in group 1 and \pm SD of $2.4 \pm 0.6$ in group 2 . The total number of attempts is 60 in group 1 and 148 in group 2. Successful anastomosis formed in 16 out of 60 treatment sites in group 1 and 60 out of 148 in group 2 .

In the 60 eyes with successful anastomosis, visual acuity improved for two or more lines in 49 eyes (82\%). Visual acuity remained stable in the rest of the patients. In the 25 eyes ( 25 patients) where anastomosis did not form, visual acuity improved for two or more lines in six eyes $(24 \%)$, decreased in nine eyes $(36 \%)$, and remained unchanged in 10 eyes. The difference between the number of eyes achieving better vision or a worsening of vision between the two groups was statistically significant $(P<0.01)$.

In all the patients with successful anastomosis, retinal haemorrhages and hard exudates started to resolve 2 weeks after treatment. Most of the retinal haemorrhages resolved 1-2 months after treatment (Figures 1 and 2). Hard exudates resolved more slowly. Cystoid macular oedema (CMO) resolved most slowly, it usually took more than half a year to resolve entirely. In one patient, $\mathrm{CMO}$ did not resolve entirely even 24 months after treatment, although retinal haemorrhages and hard exudates resolved entirely, and visual acuity improved a few months after treatment. In the 25 eyes where anastomosis did not form, retinal haemorrhages increased in five eyes (including four eyes that transformed into the ischaemic type of CRVO). Retinal haemorrhages and exudates gradually resolved in seven eyes.

Intraoperative complications occurred in most cases, including local retinal or subretinal haemorrhages in 43 eyes and slight vitreous haemorrhages in 32 eyes when the retinal vein was broken. These haemorrhages usually resolved in 4-6 weeks after treatment. Complications

Table 1 Pre-treatment characteristics of the patients with nonischaemic CRVO

\begin{tabular}{lccr}
\hline Characteristics & All eyes & Anastomosis & No anastomosis \\
\hline Number of eyes & $85(85$ patients $)$ & $60(60$ patients $)$ & $25(25$ patients $)$ \\
Age (years) (mean) & $17-70(51.0 \pm 12.2)$ & $18-70(50 \pm 12.0)$ & $17-60(51.6 \pm 12.9)$ \\
Male sex & $43(51 \%)$ & $31(52 \%)$ & $12(48 \%)$ \\
VA $\geqslant 20 / 40$ when treated & $11(13 \%)$ & $9(15 \%)$ & $2(8 \%)$ \\
VA $<20 / 200$ when treated & $16(19 \%)$ & $11(18 \%)$ & $5(20 \%)$ \\
Duration of CRVO, months (mean) & $1-24(4 \pm 3.5)$ & $1-24(4.2 \pm 3.8)$ & $1-11(3.7 \pm 2.8)$ \\
\hline
\end{tabular}

CRVO, central retinal vein occlusion; VA, visual acuity. 
after treatment occurred in six eyes of six cases (Table 2). Distal vein occlusion occurred 2 weeks after treatment at three sites in two eyes (two sites in one eye in group 1 and one site in one eye in group 2) with successful anastomosis formation and improvement of vision. Four eyes in group 2 transformed into the ischaemic type of CRVO 2-3 weeks after treatment. Panretinal argon laser photocoagulation (PRP) was tried in the four eyes at the first follow-up visit. Large neovascular fronds developed at six sites in three eyes ( $4 \%$ of sites, $5 \%$ of eyes), where FFA showed widespread retinal capillary nonperfusion and neovascular leakage. In two cases, the retinal oedema and retinal haemorrhages were so serious that it prevented complete PRP. The new vessels continued to grow, causing dens vitreous haemorrhages at about 1-2 months after treatment. Complete PRP was performed in the third case where the new vessels at the treatment site gradually subsided. In the fourth case, retinal new vessels were not detected, but neovascular glaucoma occurred 1 month after treatment. No other serious complications occurred.

\section{Comments}

The mechanism of CRVA formation is still not very clear. The anastomosis can be induced by a high-intensity argon laser, dye yellow laser, or a combination of two lasers (argon laser and Nd-YAG laser). ${ }^{1,2,3}$ The anastomosis also could form naturally after penetrating ocular injury. ${ }^{8}$ Generally speaking, the anastomosis formation usually followed an injury that involved both the retina and the choroid. This was proved by an animal experiment, which showed that CRVA could only be induced when the laser intensity was high enough to break the retinal vein and the Bruch's membrane at the same time. When the laser energy was further increased, the retinal vein and the Bruch's membrane were broken at the same time, and in some specimen, when the choroidal vein was also broken, the success rate of anastomosis formation was increased prominently. ${ }^{9}$ Combined lasers with different wavelengths may be more effective in inducing a laser injury that involves both the retina and the choroid. At first, we used two lasers in group 1, the success rate of anastomosis formation was similar to that reported in the literature (33-54\%) using the original technique. ${ }^{1,2}$ In order to increase the success rate of anastomosis formation, we started to use three lasers in group 2 since 2000 February. The success rate of anastomosis formation in group 2 was higher. Most of the doctors in the literature used a very high-intensity argon laser alone, with power levels ranging from 0.6 to $4 \mathrm{~W}^{1-6}$ Two lasers were used in some eyes to create an anastomosis (argon and YAG), ${ }^{1}$ but the author did not compare the success rate of anastomosis formation of this group of patients with that of the other patients. When combined lasers were used as in our study, less argon laser intensity was necessary to induce anastomosis formation. In group 2, the krypton red laser was used in addition to the argon laser and the YAG laser. The krypton red laser has a longer wavelength and deeper penetration. It mainly focuses on the choroid with greater sparing of the inner retinal layers, ${ }^{10}$ so it may be used to break the Bruch's membrane and choroidal vein more effectively than the argon laser. We think that we can induce retinal and choroidal injury at the treatment site more effectively by using the combined lasers with three different wavelengths. This may explain the higher success rate of anastomosis formation in group 2.

Recently, modifications of the technique were made in order to increase the success rate of anastomosis formation. Koizumi et $a l^{11}$ transected the retinal vein in addition to making a small incision in the wall and full thickness retina. They thought that transecting the retinal vein could increase successful anastomosis formation. Leonard et al ${ }^{12}$ reported $100 \%$ success rate of anastomosis formation by using medium-intensity, long-duration argon laser photocoagulation spots without puncturing the vein wall. They thought that puncturing the vein wall was unnecessary in inducing the anastomosis formation. These modifications of the technique were only reported in small case series and were not repeated by other doctors.

Table 2 Post-treatment characteristics of the patients with nonischaemic CRVO

\begin{tabular}{lccc}
\hline Characteristics & All eyes $(\mathrm{n}=85)$ & Anastomosis $(\mathrm{n}=60)$ & No anastomosis $(\mathrm{n}=25)$ \\
\hline VA improved for $\geqslant 2$ lines & $55(65 \%)$ & $49(82 \%)$ & $6(24 \%)$ \\
Final VA $\geqslant 20 / 40$ & $27(32 \%)$ & $25(42 \%)$ & $2(8 \%)$ \\
Final VA $<20 / 200$ & $12(14 \%)$ & $4(6 \%)$ & $8(32 \%)$ \\
Anastomosis formation & $60(71 \%)$ & $60(100 \%)$ & 0 \\
Progressed to ischaemic type & $4(5 \%)$ & 0 & $4(16 \%)$ \\
Neovascular complication & $4(5 \%)$ & 0 & $4(16 \%)$ \\
Distal vein occlusion & $2(2 \%)$ & $2 / 60(3 \%)$ & 0 \\
Follow-up time (months) & $6-36$ (Average12.2 \pm 9.9$)$ & $6-36($ average $11.6 \pm 9.3)$ & $6-30$ (average 13.7 \pm 11.4$)$ \\
\hline
\end{tabular}


One of the complications was distal vein occlusion at the treatment site, leaving a segmental area of retinal ischaemia and various neovascular and fibrous tissue proliferation. ${ }^{1}$ Sectoral scatter laser photocoagulation was used to prevent the growth of retinal neovascularization in case distal vein occlusion occurs at the treatment site. In comparison with other studies that did not use sectoral scatter laser photocoagulation, the success rate of anastomosis formation is higher in group 2 and indicates that sectoral scatter laser

photocoagulation did not interfere with the formation of anastomosis.

In one study, ${ }^{1}$ distal vein occlusion at the treatment site occurred in $29 \%$ of 91 eyes. In another study, ${ }^{3}$ it occurred in two out of eight cases (25\%). In the above studies, usually several overlapping argon laser shots at each treatment site were applied. In comparison, distal vein occlusion at the treatment site occurred much less frequently in our study (2\%). This can be explained as follows: the argon laser is haemoglobin-absorbing wavelength. When very high-intensity argon laser is used to break the whole layer of the retina and Bruch's membrane, it may cause thrombus formation and distal vein occlusion. As we used less argon laser intensity (1.5-2.4 W), there might have been less chance of distal vein occlusion. In comparison with the argon laser, the krypton red laser is poorly absorbed by haemoglobin, ${ }^{10}$ so it may not increase the chance of thrombus formation or distal vein occlusion at the treatment sites in group 2.

The most serious complication was neovascularization at the treatment site. The occurrence rate was reported to be about $20 \% \cdot{ }^{1-3}$ Neovascularization was usually caused by retinal ischaemia. ${ }^{1}$ In our study, neovascular complications only developed in four eyes $(5 \%)$ that transformed to the ischaemic type of CRVO without anastomosis formation. First of all, we selected patients with less degree of retinal ischaemia than that reported in other studies. ${ }^{1}$ Patients with 5-disc diameters or less of capillary nonperfusion were included in our study, whereas in most other studies, patients with 10-disc diameters or less of capillary nonperfusion were included. ${ }^{1-6}$ This may contribute to the decreased occurrence of neovascular complications in our study. Second, distal vein occlusion at the treatment site occurred much less frequently in our study as described above. This may also contribute to decreased occurrence of neovascular complications in our study. Third, during the treatment, scattered argon laser usage around and distal to the treatment site may be effective in preventing the growth of retinal neovascularization in case distal vein occlusion occurred after treatment. Sectoral scatter laser could destruct the ischaemic zones caused by distal vein occlusion. This might eliminate the source of angiogenic stimulus from the ischaemic zones. In one study, ${ }^{1}$ neovascular complications associated with distal vein occlusion occurred in almost all the cases, whereas none occurred in our study. In the two cases that transformed to the ischaemic type of CRVO, the retinal ischaemia was so widespread that sectoral scatter laser photocoagulation was not enough to prevent the development of neovascularization. Although PRP treatment was tried at the first follow-up visit, the retinal oedema and retinal haemorrhages were so serious that it prevented complete PRP. The new vessels continued to grow, because the source of angiogenic stimulus from the ischaemic zones still existed owing to incomplete PRP.

After successful anastomosis, all the patients showed improved retinal changes and most patients (82\%) had an improved visual acuity. This is in agreement with most other studies. ${ }^{1,2}$ In the natural history report of CRVO by $\mathrm{CVOS}^{13}{ }^{13}$ ischaemia developed in $34 \%$ of the 547 initially perfused eyes, whereas none occurred in our cases with successful anastomosis. This indicates that successful anastomosis may prevent the development of ischaemia.

Among the 714 eyes enrolled in the study conducted by the CVOS, ${ }^{13} 29 \%$ had an initial visual acuity of $20 / 40$ or better and $28 \%$ of the eyes were worse than $20 / 200$. After 3-year follow-up, the final visual acuity remained $20 / 40$ or better in $28 \%$ of the cases and were worse than $20 / 200$ in $41 \%$ of the cases. In our patients with successful anastomosis, nine eyes $(15 \%)$ had an initial visual acuity of $20 / 40$ or better and 11 eyes (18\%) were worse than 20/200 (Table 1). After successful anastomosis, 25 eyes (42\%) had a final visual acuity of $20 / 40$ or better and only four eyes $(6 \%)$ were worse than 20/200 (Table 2). This indicates that successful anastomosis may improve the visual prognosis of the patients with nonischaemic CRVO.

Limitations of this study include its retrospective design and a relatively small number of cases. However, the number of patients in this study is larger than that in most other studies of CRVA. To our knowledge, only one retrospective study, with 91 cases, had a larger number. ${ }^{1}$

In conclusion, the use of three laser wavelengths results in the creation of laser anastomosis and the success rate may be higher than when using two wavelengths alone.

\section{References}

1 McAllister IL, Douglas JP, Constable IJ, Yu DY. Laserinduced chorioretinal venous anastomosis for nonischaemic central retinal vein occlusion: evaluation of the complications and their risk factors. Am J Ophthalmol 1998; 126: $219-229$. 
2 Fekrat S, Goldberg MF, Finkelstein D. Laser-induced chorioretinal venous anastamosis for nonischaemic vein occlusion. Arch Ophthalmol 1998; 116: 43-52.

3 Browning DJ, Antoszyk AN. Laser chorioretinal venous anastomosis for nonischaemic central retinal vein occlusion. Ophthalmology 1998; 105: 670-679.

4 Browning DJ, Rotberg MH. Vitreous haemorrhage complicating laser-induced chorioretinal anastomosis for central retinal vein occlusion. Am J Ophthalmol 1996; 122: 588-589.

5 Eccarius SG, Moran MJ, Siingsby JG. Choroidal neovascular membrane after laser-induced chorioretinal anastomosis. Am J Ophthalmol 1996; 122: 590-591.

6 Jeffrey KL. Epiretinal membrane and traction retinal detachment complicating laser-induced chorioretinal venous anastomosis. Am J Ophthalmol 1997; 123: 698-699.

7 Central Vein Occlusion Study Group. Baseline and early natural history report: the central vein occlusion study. Arch Ophthalmol 1993; 111: 1087-1095.

8 Goldberg MF. Chorioretinal vascular anastomosis after blunt trauma to the eye. Am J Ophthalmol 1978; 85: 171-173.
9 Vijayasekaran S, Yu DY, McAllister I, Barry C, Constable I. Optimal conditions required for the creation of an iatrogenic chorioretinal venous anastomosis in the dog using argon green laser photocoagulation. Curr Eye Res 1995; 14: 63-70.

10 Marshall J, Bird AC. A comparative histopathological study of argon and krypton laser of the human retina. $\mathrm{Br} J$ Ophthalmol 1979; 63: 657-668.

11 Koizumi K, Nishiura M, Yamamoto T, Mchida T, Nakamura $\mathrm{T}$, Ouchi $\mathrm{M}$ et al. Intentional complete interruption of a retinal vein after vitrectomy might improve the rate of successful chorioretinal venous anastomosis formation in central retinal vein occlusion. Graefes Arch Clin Exp Ophthalmol 2002; 240: 787-794.

12 Leonard BC, Coupland SG, Kertes PJ, Bate R. Long-term follow-up of a modified technique for laser-induced chorioretinal venous anastomosis in nonischaemic central retinal vein occlusion. Ophthalmology 2003; 110: $948-954$

13 The Central Vein Occludon Study Group. Natural history and clinical management of central retinal vein occlusion. Arch Ophthalmol 1997; 115: 486-491. 